Audiology

Neurotology
Audiol Neurotol 2013;18:247-260

DOI: $\underline{10.1159 / 000351302}$
Received: July 9, 2012

Accepted after revision: March 29, 2013

Published online: July 20, 2013

\title{
Using Temporal Modulation Sensitivity to Select Stimulation Sites for Processor MAPs in Cochlear Implant Listeners
}

\author{
Soha N. Garadat ${ }^{a}$ Teresa A. Zwolan ${ }^{b}$ Bryan E. Pfingst ${ }^{a}$ \\ ${ }^{a}$ Kresge Hearing Research Institute and ${ }^{b}$ Hearing Rehabilitation Center, Department of Otolaryngology, \\ University of Michigan, Ann Arbor, Mich., USA
}

\section{Key Words}

Cochlear implantation - Across-site differences .

Speech recognition - Modulation detection thresholds .

Personalized processor fitting

\begin{abstract}
Previous studies in our laboratory showed that temporal acuity as assessed by modulation detection thresholds (MDTs) varied across activation sites and that this site-to-site variability was subject specific. Using two 10-channel MAPs, the previous experiments showed that processor MAPs that had better across-site mean (ASM) MDTs yielded better speech recognition than MAPs with poorer ASM MDTs tested in the same subject. The current study extends our earlier work on developing more optimal-fitting strategies to test the feasibility of using a site-selection approach in the clinical domain. This study examined the hypothesis that revising the clinical speech processor MAP for cochlear implant $(\mathrm{Cl})$ recipients by turning off selected sites that have poorer temporal acuity and reallocating frequencies to the remaining electrodes would lead to improved speech recognition. Twelve $\mathrm{Cl}$ recipients participated in the experiments. We found that site selection procedure based on MDTs in the presence of a masker resulted in improved performance on
\end{abstract}

\section{KARGER}

E-Mail karger@karger.com

www.karger.com/aud consonant recognition and recognition of sentences in noise. In contrast, vowel recognition was poorer with the experimental MAP than with the clinical MAP, possibly due to reduced spectral resolution when sites were removed from the experimental MAP. Overall, these results suggest a promising path for improving recipient outcomes using personalized processor-fitting strategies based on a psychophysical measure of temporal acuity.

(c) 2013 S. Karger AG, Basel

\section{Introduction}

Psychophysical experiments with cochlear implant (CI) subjects often reveal considerable variability in the overall benefit received from cochlear implantation [Munson and Nelson, 2005; Gfeller et al., 2008; Wilson and Dorman, 2008]. Over the years, increased attention has been devoted to capturing and overcoming some of the limiting factors with the hope of achieving maximal benefit from electrical stimulation and more uniform performance across CI users. While the overall success of cochlear implantation has increased, variability in performance, particularly in difficult listening conditions, still poses a significant challenge in the field. Local irregulari-
(C) 2013 S. Karger AG, Basel

1420-3030/13/0184-0247\$38.00/0
Soha N. Garadat

Department of Hearing and Speech Sciences

University of Jordan

11942 Amman (Jordan)

E-Mail s.garadat@ju.edu.jo 
ties within listeners in the electrode-neuron interface and the properties of the activated neurons could underlie some of this variability.

Consistent with this notion, large differences in responses to electrical stimulation using various psychophysical measures have been reported across stimulation sites within individual listeners [e.g. Pfingst and Xu, 2004; Bierer, 2007; Nelson et al., 2008; Pfingst et al., 2008; Bierer and Faulkner, 2010; Garadat and Pfingst, 2011; Jones et al., 2013]. Nonetheless, to date, an area that remains poorly addressed is the effect of site-specific acuity on overall performance. The fact that performance differs from one stimulation site to another in individual implants suggests that not all sites of stimulation contribute equally to overall performance and that stimulation at poorly performing sites might be detrimental to the overall clinical outcome. This raises the important question of whether removing suboptimal sites would lead to improvement in overall performance. In order to effectively utilize the across-site variation in performance for selection of stimulation sites, it is important to characterize differences in performance across sites of stimulation using a measure that is predictive of speech perception.

An important feature of speech is its variation in amplitude as a function of time. The low frequency amplitude fluctuations, which have been referred to as the temporal envelope, have been shown to convey linguistic information about manner of articulation, voicing and vowel identity [Van Tasell et al., 1987; Rosen, 1992]. This is relevant to ways in which multichannel implants function where a temporal code is used. Typically, the envelopes of the filtered speech sounds are extracted and used to amplitude-modulate interleaved trains of biphasic pulses [Wilson et al., 1991; Skinner et al., 2002]. In electrical hearing, envelope cues contribute significantly to speech recognition [Shannon et al., 1995; Fu, 2002] given that CI recipients typically have poor spectral resolution [Friesen et al., 2001]. Consequently, the extent to which CI recipients are able to resolve temporal envelope information might predict the overall outcome of cochlear implantation.

One commonly used measure of temporal resolution is the modulation detection threshold (MDT). This measure can be useful as it might relate to the perception of amplitude variation in speech such that CI users with good speech recognition would exhibit greater sensitivity for amplitude modulation than those with poor speech recognition. Indeed, previous studies have revealed such a relation between MDTs measured on a single channel and speech recognition [Fu, 2002; Luo et al., 2008]. In multichannel CIs, however, temporal cues underlying speech recognition are more complex than those measured with a single site due to channel interaction. Modern CIs use arrays with multiple electrodes specifically designed to take advantage of the natural tonotopic organization of the cochlea and the pattern of auditory nerve innervations. For optimal stimulation, channel independence needs to be achieved to selectively activate the targeted neural population along the electrode array with appropriate temporal information. However, channel independence is rare and evidence of interaction among channels is widely reported [Chatterjee and Shannon, 1998; Cohen et al., 2003; Eisen and Franck, 2005; Bierer, 2007]. Interaction among channels has been shown to interfere with the extent to which CI recipients have been able to resolve temporal information [Richardson et al., 1998; Chatterjee, 2003] and to also understand speech [Chatterjee and Shannon, 1998; Throckmorton and Collins, 1999; Boëx et al., 2003].

In order to identify site-to-site variability in electrical stimulation, it is important to select a measure that examines temporal acuity in the context of channel interaction. Previous work [Garadat et al., 2012] showed this variability can be identified using MDTs measured in the presence of interleaved unmodulated pulse trains presented on the adjacent site. Using 10-channel MAPs, the study further showed that by selecting sites that had the best masked MDTs, better speech recognition was achieved than when sites with worst masked MDTs were selected. Using the same MDT data that were collected in the study by Garadat at al. [2012], the current work expands this previous work by translating its research components into the clinical domain. The goals of this current work were: (1) to identify differences in MDTs across the electrode array using a masked MDT measure, and (2) to use these differences to construct a subject-specific personalized experimental MAP that was akin to the subjects' everyday MAP but eliminates stimulation to selected sites with poor performance from each tonotopic region. It was hypothesized that using patient-specific fitting procedures that identified the local conditions across the electrode array and removed sites with poor performance would lead to better speech recognition.

\section{Materials and Methods}

In general, these experiments used the same subjects, stimuli and equipment as those reported in the previous work [Garadat et al., 2012]. The MDT data used in these experiments are essentially the same reported in the paper by Garadat et al. [2012], but the 
Table 1. Subject demographics

\begin{tabular}{|c|c|c|c|c|c|c|c|c|c|}
\hline Subject & Gender & $\begin{array}{l}\text { Age, } \\
\text { years }\end{array}$ & $\begin{array}{l}\text { Age at onset } \\
\text { of deafness, } \\
\text { years }\end{array}$ & $\begin{array}{l}\text { Duration } \\
\text { of deafness, } \\
\text { years }\end{array}$ & $\begin{array}{l}\text { Duration } \\
\text { of CI use, } \\
\text { years }\end{array}$ & $\begin{array}{l}\text { Mode of } \\
\text { stimulation }\end{array}$ & $\begin{array}{l}\text { Implant } \\
\text { type }\end{array}$ & Strategy & $\begin{array}{l}\text { Etiology } \\
\text { of deafness }\end{array}$ \\
\hline S60 & $\mathrm{M}$ & 70 & 60 & 10 & 7 & Bilateral & $\mathrm{CI} 24 \mathrm{R}(\mathrm{CS})$ & $\mathrm{ACE}$ & Hereditary \\
\hline S67 & $\mathrm{M}$ & 69 & 59 & 10 & 10 & Unilateral & CI24R(CS) & $\mathrm{ACE}$ & Hereditary \\
\hline S69 & $\mathrm{M}$ & 69 & 60 & 9 & 5 & Bilateral & $\mathrm{CI} 24 \mathrm{M}$ & $\mathrm{ACE}$ & Noise induced \\
\hline S81 & $\mathrm{F}$ & 59 & 52 & 7 & 5 & Bilateral & CI24RE(CA) & $\mathrm{ACE}$ & Hereditary \\
\hline S82 & $\mathrm{F}$ & 60 & 36 & 24 & 6 & Unilateral & CI24RE(CA) & ACE & Unknown \\
\hline S83 & $\mathrm{F}$ & 65 & 13 & 52 & 6 & Unilateral & CI24R(CS) & ACE & Otosclerosis \\
\hline S84 & M & 51 & 26 & 25 & 5 & Unilateral & CI24RE(CA) & $\mathrm{ACE}$ & Hereditary \\
\hline S85 & $\mathrm{F}$ & 63 & 30 & 33 & 4 & Unilateral & CI24RE(CA) & $\mathrm{ACE}$ & Hereditary \\
\hline S86 & $\mathrm{F}$ & 64 & 60 & 4 & 3 & Bilateral & CI24RE(CA) & $\mathrm{ACE}$ & Hereditary \\
\hline
\end{tabular}

experimental processor MAPs and processor settings were much closer to those used in the subjects' normal clinical devices.

\section{Listeners}

Twelve postlingually deaf subjects (6 males and 6 females) with various types of Nucleus cochlear implants participated in this study. One subject had a CI24M (straight array) implant, 5 had CI24R (Contour) implants and 6 had CI24RE (Freedom ${ }^{\circledR}$ ) implants. Subjects ranged from 51 to 75 years of age. All subjects had at least 12 months of experience using their CI. Subjects' demographics are given in table 1 . Testing was completed in two 8-hour sessions with frequent breaks during testing. All subjects were compensated for their participation. The use of human subjects in this study was reviewed and approved by the University of Michigan Medical School Institutional Review Board.

\section{Psychophysical Procedures}

Electrical Stimulation Hardware

All testing was conducted inside a large, carpeted, doublewalled, sound-attenuating chamber to maintain consistency in the testing environment across sessions and subjects. Stimuli for the psychophysical tasks were delivered directly to the subject's implanted receiver/stimulator by software that controlled the external processor. All communication with the processor was controlled using custom software run on a PC. Sequences of frames were generated and sent to the processor via Cochlear NIC II (Nucleus Implant Communicator) software [Swanson, 2004]. All testing was conducted using a laboratory Freedom speech processor (Cochlear Corporation, Englewood, Colo., USA) which was used in place of the subject's processor in order to assure uniformity in the external hardware. No acoustic stimulation was used for the psychophysical tests.

Monopolar stimulation (MP $1+2)$ was used for all measurements. The term 'stimulation site' is used in this study to indicate where stimulation was delivered along the electrode array. For monopolar stimulation, the stimulation site corresponds to the number of the scala tympani electrode, where the most basal electrode was number 1 and the most apical electrode was number 22 .
Measurements of T and C Levels

Cochlear Custom Sound Suite 3.1 software was used to obtain absolute detection thresholds (T levels) and maximum comfortable loudness levels (C levels). The stimulus burst duration was 500 $\mathrm{ms}$ with phase duration of $50 \mu$ s presented at a rate of $900 \mathrm{pps}$ and $200 \mathrm{~ms}$ in between bursts. T and C levels were determined for all electrodes as is typically done in clinical implant fitting. Specifically, T levels were obtained for all electrodes using an adjustment method until the subjects were just able to detect a soft sound on each electrode. Subsequently, C levels were obtained by increasing the level of stimulation for each electrode until the subject indicated the stimulus was 'loud but comfortable' and could be listened to 'for long periods of time'. Using an adjacent-reference loudness balance design described by Zwolan et al. [1997], all stimulation sites were loudness balanced. Dynamic range (DR) for each site was calculated by obtaining the difference between the $T$ level and the $\mathrm{C}$ level of the respective site.

\section{Modulation Detection Thresholds}

As reported in the previous experiment [Garadat et al., 2012], MDTs were obtained for each site in the subjects' electrode arrays in the presence of an interleaved unmodulated masker (masked MDTs). The masker was presented on the adjacent more-apical site for all sites with the exception of site 22, where the masker was on the adjacent more-basal site. Stimuli were $500 \mathrm{~ms}$ trains of symmetric biphasic pulses with a mean phase duration of $50 \mu$ s and an interphase interval of $8 \mu$ s presented at a rate of $900 \mathrm{pps}$ and a presentation level equal to $50 \%$ of the DR using custom software. Pulse duration, rather than current amplitude, was modulated because finer control of stimulus charge could be achieved using pulse duration. The phase duration of the pulse was modulated by a $10-\mathrm{Hz}$ sinusoid that started and ended at zero phase. The positive and negative phases of the pulses were modulated equally to maintain charge balance and the interphase interval was held constant. The modulation index $(m)$ was defined as

$$
m=\left(\mathrm{PD}_{\max }-\mathrm{PD}_{\min }\right) /\left(\mathrm{PD}_{\max }+\mathrm{PD}_{\min }\right),
$$


where $\mathrm{PD}_{\max }$ and $\mathrm{PD}_{\min }$ are the maximum and minimum phase durations, respectively. We report modulation values in $\mathrm{dB}$ re $100 \%$ modulation (i.e. $20 \log m$ ).

A four-alternative forced choice paradigm was used to determine MDTs at $50 \%$ of the DR. On each trial, subjects were presented with four sequential stimuli that were $500 \mathrm{~ms}$ in duration, separated by $500 \mathrm{~ms}$ intervals and indicated by boxes on the computer screen. One of the four intervals was randomly chosen on each trial to contain the modulated signal while the remaining three intervals contained identical unmodulated pulse trains. Subjects were instructed to choose the interval that sounded different from the other three intervals. Feedback was provided after each trial by marking the chosen interval as ' $\mathrm{C}$ ' for a correct response or ' $\mathrm{X}$ ' for an incorrect response. A two-down, one-up adaptive-tracking procedure [Levitt, 1971] was used, starting with a modulation depth of $50 \%$. Modulation depth was increased or decreased in steps of $6 \mathrm{~dB}$ for the first 2 reversals, $2 \mathrm{~dB}$ for the next 2 reversals and $1 \mathrm{~dB}$ for the next 8 reversals. Each run was terminated after 12 reversals were obtained. MDTs were defined as the average of the modulation depths at the last 8 reversals.

Using this procedure, detection thresholds for depth of modulated pulse duration were determined in the presence of an unmodulated masker for all stimulation sites in the subjects' electrode arrays in a random order. The procedure was repeated twice using a different randomization so that a total of three MDTs were obtained for each site. The average of these three values was used as the estimate of the MDT for each site.

\section{Speech Processor MAPs}

The experimental speech processor was mapped using approaches aimed at improving mean modulation sensitivity in comparison to the subjects' everyday MAPs. As a general rule, the experimental MAP used all stimulation sites available in the subjects' everyday MAPs except that 5 sites which had the highest (poorest) masked MDTs were turned off and filter bandwidths were adjusted accordingly. In order to avoid removal of large numbers of sites from particular area(s) of the array, the electrode array was divided into 5 segments and usually only one site per segment was deactivated in order to maintain the full range of place-pitch representation. Given that there are 22 electrodes in the electrode array, the electrode-to-segment assignment was uneven when there were more than 20 electrodes enabled. Specifically, some segments had five stimulation sites and others had four stimulation sites per segment; these were selected based on the goal of maximizing the diversity in performance within segments. In addition, if there was a segment that had overall good modulation sensitivity relative to the rest of the electrode array, no stimulation sites were turned off from that segment and instead an additional stimulation site was turned off in a segment that had more than one poorly performing site. However, no more than two adjacent sites were turned off. An example of site selection is illustrated in figure 1.

The experimental MAP was similar in all aspects (e.g. processing strategy, pulse duration and pulse rate) to the clinical MAP except for the 5 stimulation sites that were disabled. The frequency-to-electrode assignment was automatically adjusted by the clinical software such that the frequency spectrum range remained the same but the bands were broadened to reallocate the frequency information originally processed in the disabled electrodes. SmartSound options were the same for the two MAPs and the same as those that the subjects used daily.

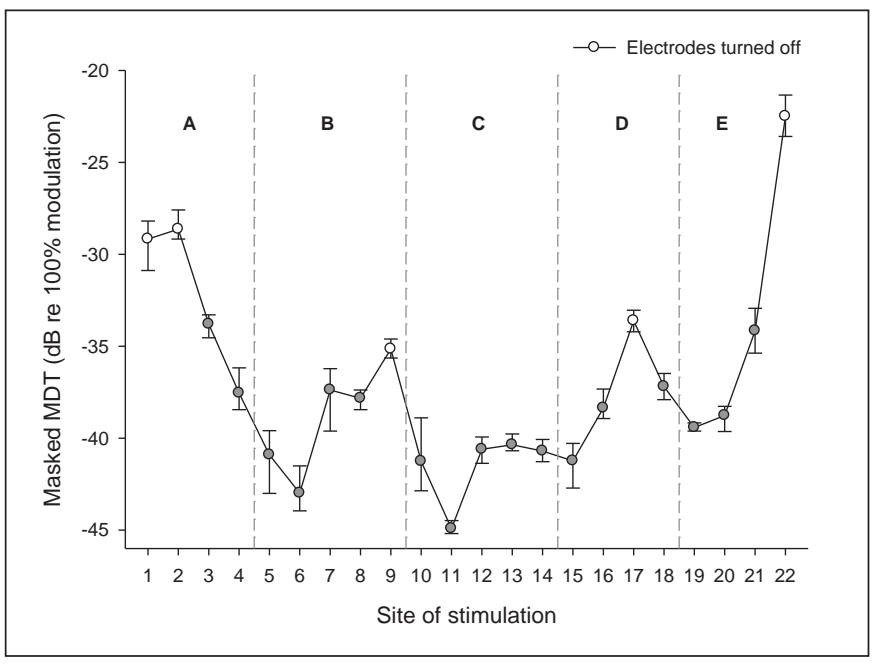

Fig. 1. Masked MDTs for one subject are displayed to demonstrate the site-selection approach used to construct the experimental MAP. For each site, the mean MDT and the range for three repeated measures are shown. The electrode array was divided into 5 segments as shown (labeled A-E). Disabled sites are marked with open symbols. In general, one site with the worst masked MDTs was disabled from each segment, but there were exceptions as noted in the text. In this example, there were no poorly performing sites in segment $\mathrm{C}$, so two sites were disabled in segment $\mathrm{A}$.

Prior to the data collection, subjects were instructed to adjust the volume setting on their processor to a comfortable level while listening to sentences from the hearing in noise test (HINT) list presented in quiet and in noise; these sentences were not reused during speech testing. Several volume settings were tried with the everyday MAP and the experimental MAP until subjects found a setting that was comfortable and judged to be equally loud across the two MAPs. Although this produced different volume settings across listeners, it was felt necessary to not restrict all subjects to a specific volume setting for the following reasons: (1) it might introduce differences in performance due to reduced audibility or uncomfortably loud perception; (2) differences in loudness perception are known to impact performance on speech measures and probably introduce further differences between MAPs and, therefore, by setting the volume to a comfortable level but equally loud for each subject would allow us to limit this problem and to capture any real differences in speech perception across the two MAPs, and (3) in real life, patients do not use fixed volume setting and rather they freely adjust their volume to optimize their listening experience to compensate for any listening difficulty. After determining the appropriate volume setting based on listening with both MAPs, the subjects were then instructed to maintain this setting throughout the testing session. Therefore, different volume settings were used across subjects but the volume setting used by a given subject was the same for the two MAPs.

Speech Measurements

All speech measurements were conducted in a carpeted double-walled sound booth (Acoustic Systems Model RE 242S). 
Speech measures consisted of medial vowel and consonant discrimination, and sentence recognition in quiet and in noise. Speech-shaped noise from the HINT was used when speech recognition was evaluated in the presence of an acoustic masker. Stimuli were passed through a Rane ME60 graphic equalizer and a Rolls RA235 35W power amplifier and presented through a loudspeaker positioned $1 \mathrm{~m}$ away from the subject at $0^{\circ}$ azimuth. All target stimuli were presented at a fixed intensity level of $60 \mathrm{~dB}$ SPL with the exception of sentences in noise where an adaptive procedure was used as detailed below. Speech levels were calibrated with a sound-level meter (type 2231; Brüel and Kjær, Naerum, Denmark). The sound-level meter microphone was positioned $1 \mathrm{~m}$ away and at $0^{\circ}$ azimuth. A slow time setting and an ' $A$ ' frequency weighting scale were used for the calibration.

Vowel stimuli [Hillenbrand et al., 1995] consisted of 12 medial vowels presented to subjects with custom software in an /h/-vowel/d/ context (heed, hawed, head, who'd, hid, hood, hud, had, heard, hoed, hod, hayed). Using a 12-alternative forced-choice paradigm, subjects were instructed to click on the appropriate token on a computer screen to indicate their response selection. The 12 vowel tokens were alphabetically arranged and presented in a grid on the computer screen. Two male talkers (No. 48 and No. 49) and two female talkers (No. 39 and No. 44) were selected from the recordings yielding a total of 48 stimuli per run. Stimuli were presented in quiet and with four different signal-to-noise ratios (SNRs) of $+15,+10,+5$ and $0 \mathrm{~dB}$. Tokens were presented in random order without replacement. Chance level on this test was $8.33 \%$ correct.

Consonant stimuli [Shannon et al., 1999] consisted of twenty naturally spoken American English consonants presented in a consonant-/a/ context (ba, cha, da, fa, ga, ja, ka, la, ma, na, pa, ra, sa, sha, ta, tha, va, wa, ya and za). Two talkers (Male No. 3 and Female No. 3) were used for a total of 40 presentations. Stimuli were presented in quiet and at SNRs of $+15,+10,+5$ and $0 \mathrm{~dB}$. A 20 -alternative forced-choice paradigm was used and subjects were instructed to click on one of the 20 choices on a computer screen to indicate their response. Chance level for the consonant test was $5 \%$ correct.

Recognition of words in sentences was measured in quiet using the HINT sentences [Nilsson et al., 1994] and in noise using City University of New York (CUNY) sentences. Subjects were instructed to listen to each sentence and then repeat it verbally. The HINT sentences were obtained from the Minimum Speech Test Battery for Adult Cochlear Implant Users (House Ear Institute and Cochlear Corporation, 1996). Lists of 10 sentences were drawn at random without replacement from the 25 lists in the test corpus so that a different list was used for each repetition. A score was calculated based on the percentage of the words correctly repeated per list.

For the CUNY sentences in noise, an adaptive procedure was used to measure reception thresholds for sentences (RTS) in the presence of speech-shaped noise. RTS is defined as the SNR needed to achieve $50 \%$ sentence intelligibility. Each sentence was scored by the experimenter as correct if the subject successfully repeated all of the words in a given sentence or incorrect if the subject missed any of the words in the sentence. Based on the accuracy of the subject's response, the SNR was decreased (following a correct response) or increased (following an incorrect response) in $2 \mathrm{~dB}$ steps. The peak level of the combined signal (sentences plus noise) was held constant at $60 \mathrm{~dB}$ SPL while the SNR changed from trial to trial. As noted above, the subjects were instructed to adjust their speech processor to a comfortable listening level prior to data collection and to not change the setting during testing. All adaptive tracks were started at an easy SNR of $20 \mathrm{~dB}$ and were completed after 10 reversals were obtained. RTS was calculated as the average of the SNRs at the last 6 reversals.

All speech stimuli for the different speech measures were presented a single time only and feedback was not provided. Each test was presented two times and the results of the two different lists were averaged to yield speech recognition scores. Training was provided prior to the data collection for each measure. The training session for the consonant and vowel recognition tasks consisted of a preview of the stimuli followed by two practice runs with feedback in quiet and two runs in noise at an SNR of $5 \mathrm{~dB}$. For measures of sentence recognition, training was provided in the form of two runs in quiet and two runs in noise using a new list each time; feedback was not provided.

\section{Results}

Figure 2 shows MDT results obtained in the presence of a masker presented on the adjacent site for the $12 \mathrm{CI}$ participants; data from each participant are shown in a separate panel. These data are essentially the same as those reported in the previous work by Garadat et al. [2012]. As reported previously, there was considerable variation in modulation sensitivity across sites of stimulation as well as across listeners. For each listener, MDTs were averaged across stimulation sites to facilitate comparison across listeners and in relation to other measures. This is referred to as across-site mean (ASM), which is reported in the lower right corner of each panel. Additionally, to characterize across-site variation for each listener, across-site variance (ASV) was calculated and is also reported in figure 2 .

To examine differences in modulation sensitivity for the everyday MAP and the experimental MAP, ASM and ASV were calculated for each subject by averaging across the stimulation sites used in the subjects' everyday MAPs (all available sites) and those used to construct the experimental MAP (selected sites); results are shown in figure 3. Data were compared using one-way repeated measures ANOVA with MAP (experimental vs. everyday) as the within-subject factor. As expected, the experimental MAP had significantly better overall ASM modulation sensitivity in the presence of a masker $[F(1,11)=63.396$, $\mathrm{p}<0.0001$ ] than that obtained with the subjects' everyday MAPs. However, the analyses showed that there were no statistically significant differences between the two MAPs for ASVs. 


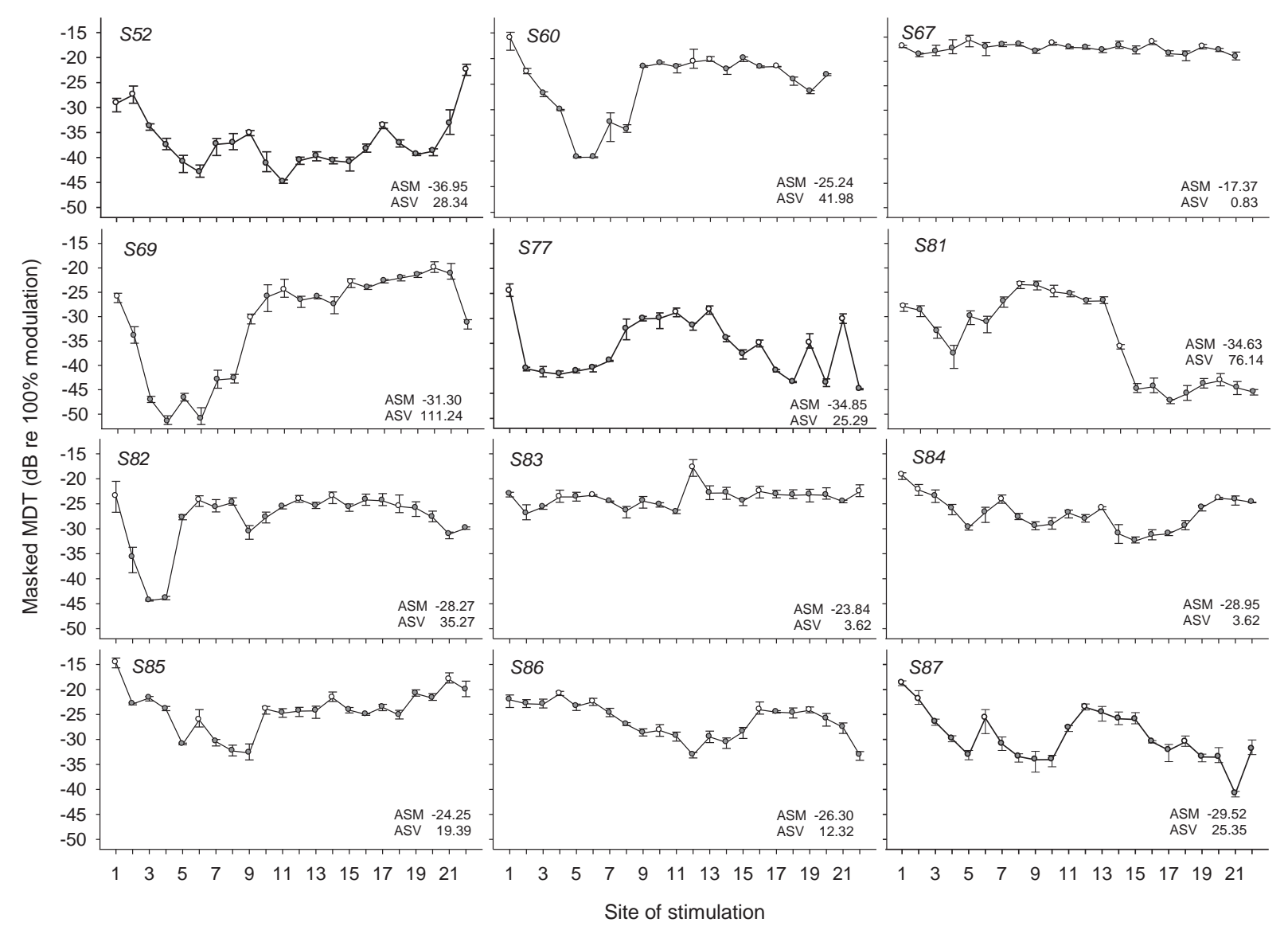

Fig. 2. Masked MDT means and ranges are shown as a function of stimulation site. Each panel represents data from one listener. Open symbols represent sites that were turned off in order to create the experimental MAP. ASM and ASV were calculated for each subject and are displayed in the lower right corner of each panel.

In order to determine the effect of overall temporal acuity on speech recognition, the two MAPs were compared for the various speech-recognition measures. Initially, it was important to examine the effect of certain variables such as everyday listening mode (unilateral, bilateral) and difference in volume setting across subjects on the current results. For example, it was important to control for the listening mode that subjects used daily to account for the concern when a bilateral user is forced to listen with one CI. In addition, given that it was necessary to conduct the speech tests at a comfortable listening level for each listener, this resulted in several volume control settings $(5,7,8$ or 9$)$. Therefore, the results from each of the four speech measurements were initially subjected to a mixed ANOVA. Specifically, data obtained from the sentence recognition measures were subjected to a mixed
ANOVA with one within-subject factor (MAP) and two between-subject factors (everyday listening mode and volume setting). In contrast, The data obtained from the consonants and vowels were subjected to a two-way mixed ANOVA with two within-subject factors (MAP, SNR) and two between-subject factors (everyday listening mode and volume setting). None of the analyses showed that everyday listening mode and/or volume settings of the processor were predictors of performance on the speech measures, indicating that there were no reliable differences in performance between the two MAPs due to these factors. However, the important factor of MAP was significant for consonants $[F(1,5)=11.521$, $\mathrm{p}<0.05]$ and for CUNY sentences in noise $[\mathrm{F}(1,5)=$ $49.748, \mathrm{p}<0.01]$ where performance was better with the experimental MAP than that with the everyday MAP. For 


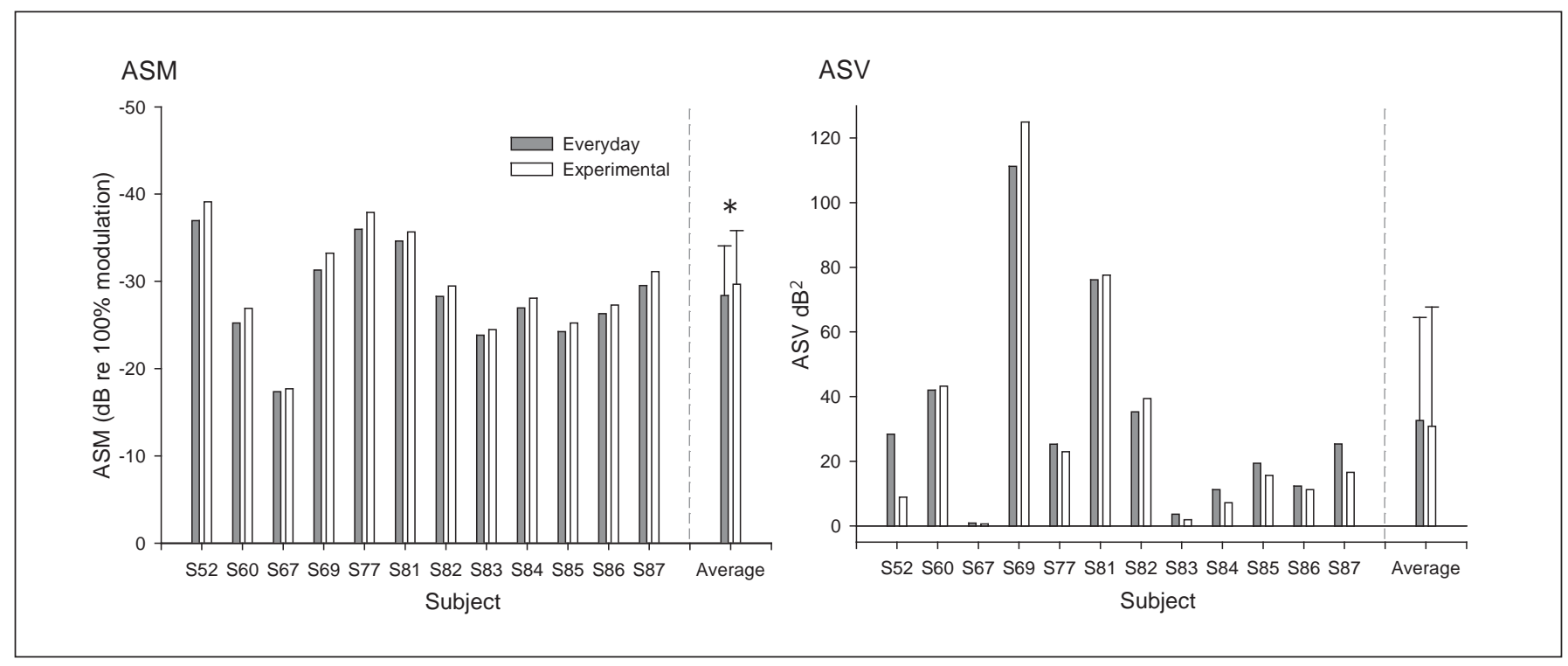

Fig. 3. Averaged ASM and ASV (+SD) along with the individual subject data for masked MDTs are compared for the everyday and the experimental MAPs. An asterisk represents a statistically significant difference between the two MAPs.

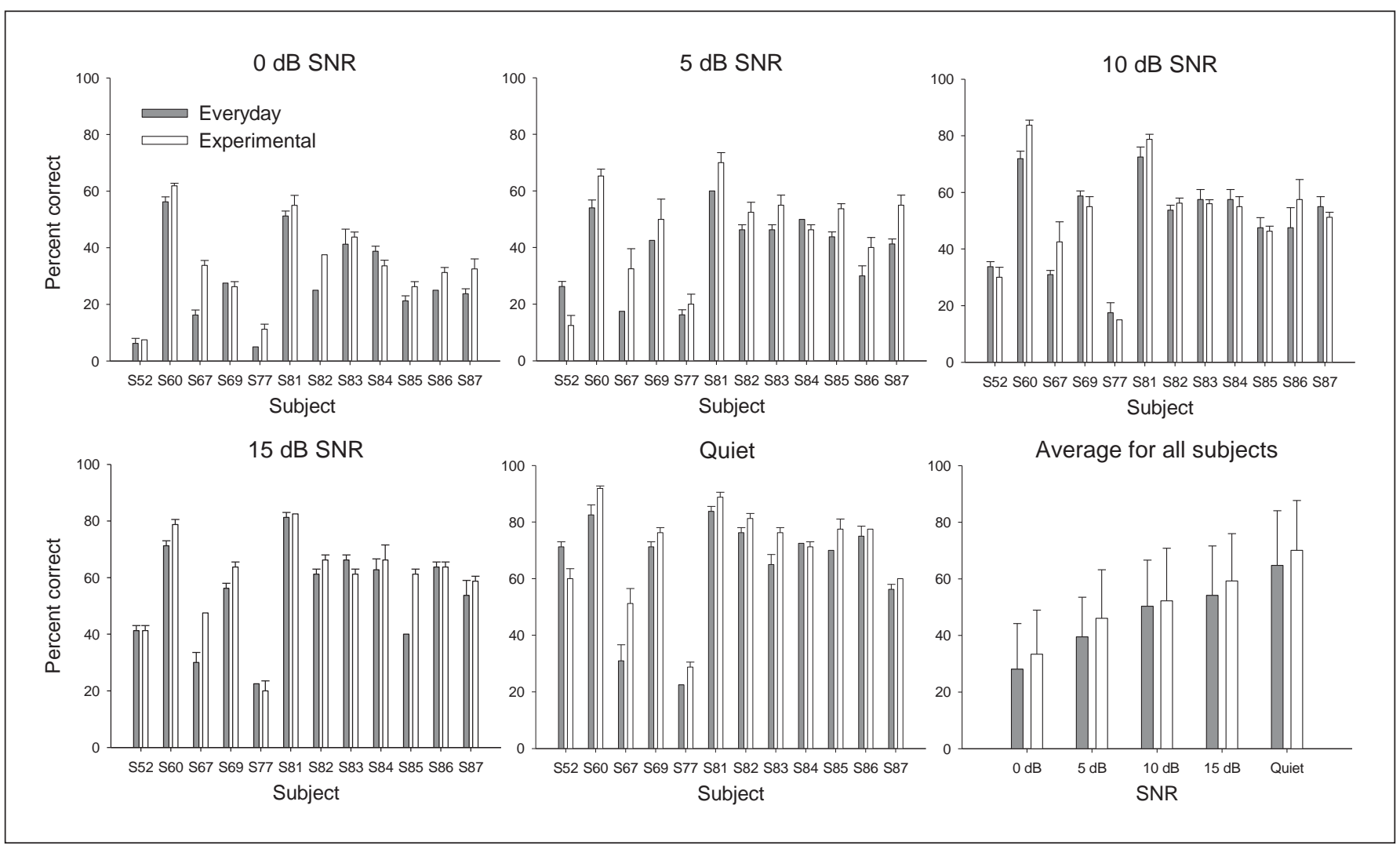

Fig. 4. Individual consonant discrimination percent scores (mean $+\mathrm{SD})$ are compared for the everyday and the experimental MAPs. The different SNR conditions are represented in different panels. Averaged scores across listeners $(+\mathrm{SD})$ as a function of SNR are displayed in the bottom right panel. 


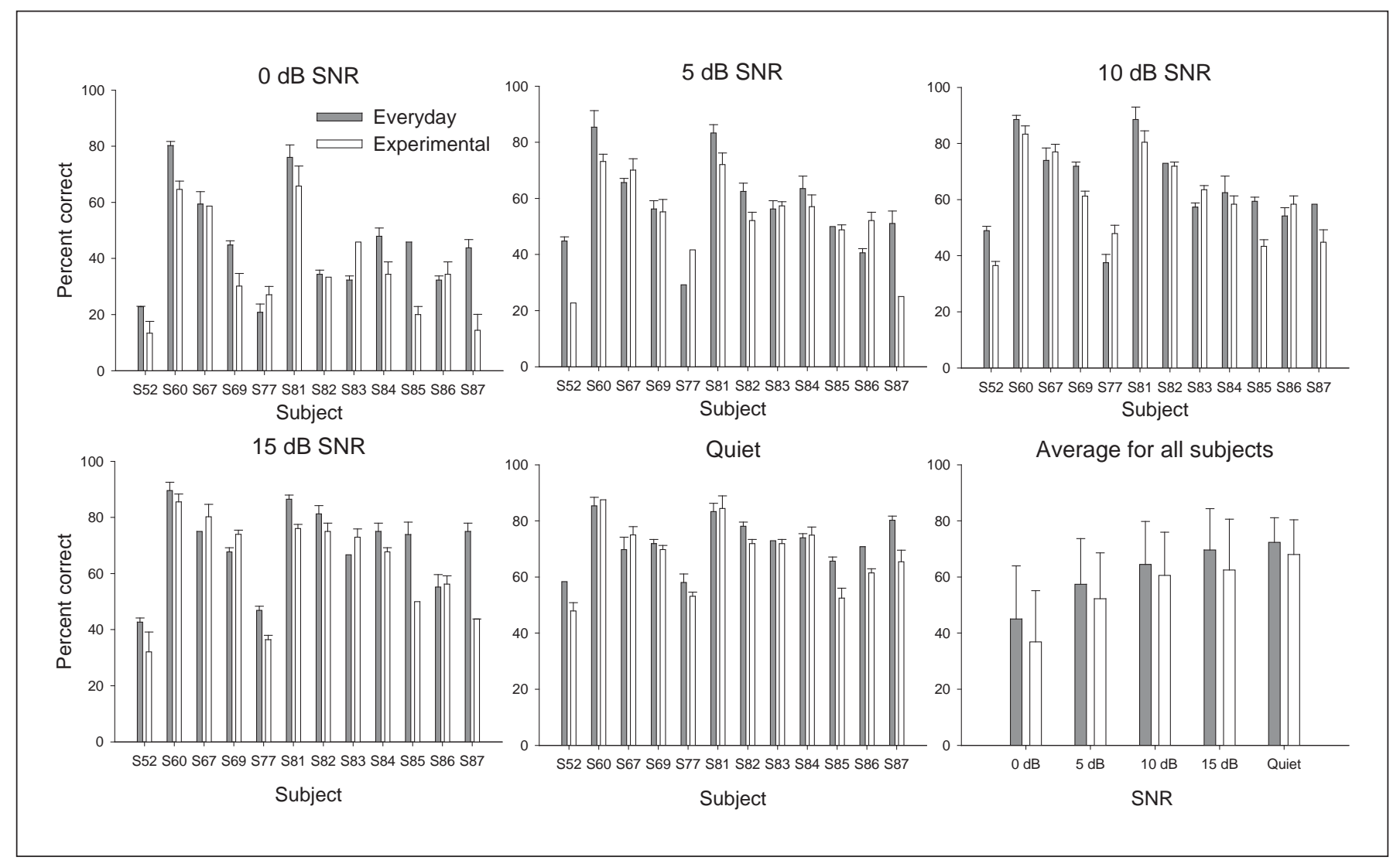

Fig. 5. Individual vowel discrimination percent scores (mean $+\mathrm{SD})$ are compared for the everyday and the experimental MAPs in different panels. The different SNR conditions are represented in different panels. Averaged scores across listeners (+SD) as a function of SNR are displayed in the bottom right panel.

the vowel recognition measure, listeners did better with the everyday MAP than with the experimental MAP [F (1, $5)=8.319, \mathrm{p}<0.05]$ and there were no differences in performance on the HINT sentences measure $(p>0.05)$. Given these results, the two within-subject factors were removed and the data analyses were repeated using a twoway repeated measure ANOVA with two within-subject factors. The independent variables were MAP (everyday and experimental) and SNR $(0,+5,+10,+15$ and quiet). The results of these analyses are described below.

Results of the consonants in quiet and at SNRs of $0,+5$, +10 and +15 are compared for the two MAPs and are shown in figure 4 . The individual data for the 12 participants along with their average scores in percent correct are displayed in the different panels for each listening condition. Analyses indicated that, on average, consonant discrimination scores were significantly better with the experimental MAP than with the everyday MAP [F (1,
$11)=9.211, \mathrm{p}<0.05]$. Results further revealed that the effect of SNR was also significant $[\mathrm{F}(4,11)=63.837, \mathrm{p}<$ $0.0001]$. The interaction between MAP and SNR was not significant $(p>0.05)$. Follow-up tests were conducted to evaluate mean differences in scores as a function of SNR; the Bonferroni procedure was used to control for type I error. Post hoc pairwise $t$ tests showed that consonant discrimination scores continuously improved with increasing SNR $(\mathrm{p}<0.01)$.

Similar analyses were conducted for the vowel discrimination scores; results are shown in figure 5. In contrast to the consonant results, statistical analyses revealed that overall vowel discrimination was significantly better with the everyday MAPs compared to performance with the experimental MAPs $[\mathrm{F}(1,11)=5.471, \mathrm{p}<0.05]$. As expected, results further revealed that the effect of SNR was significant $[\mathrm{F}(4,11)=59.808, \mathrm{p}<0.0001]$. There was no significant interaction between MAP and SNR ( $p>$ 


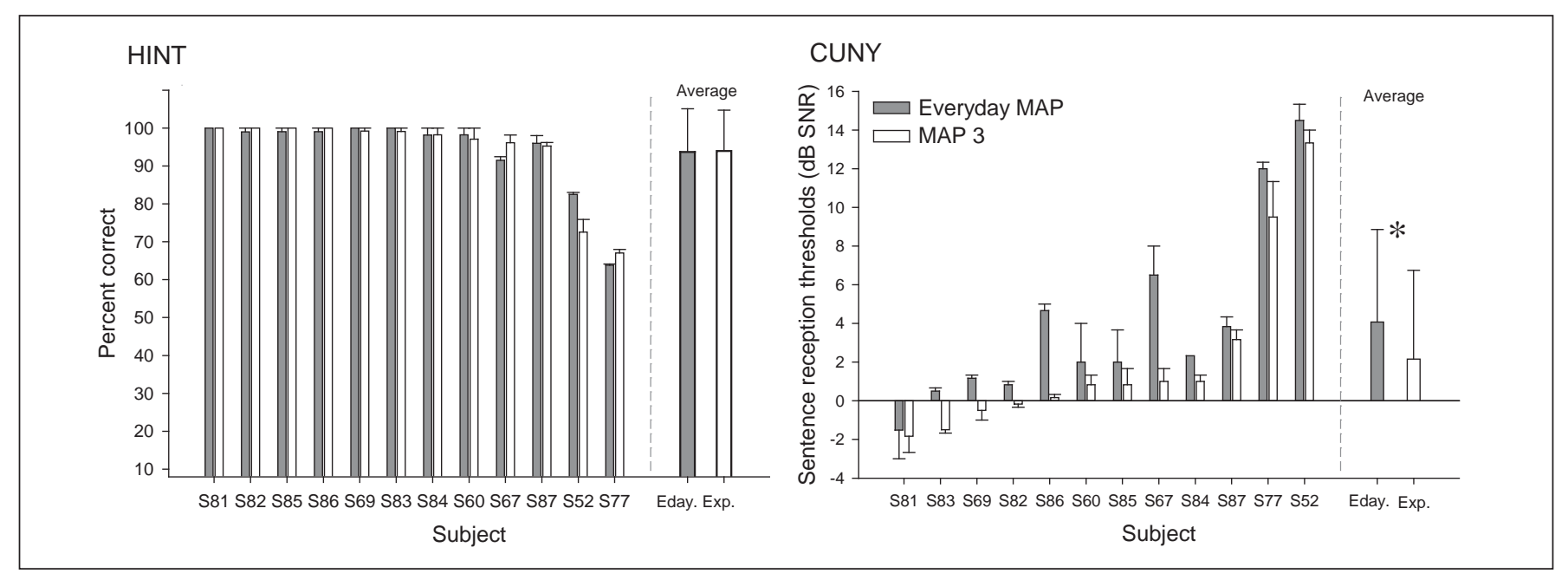

Fig. 6. Listeners' performance on sentence discrimination tasks is shown for HINT sentences in quiet (left panel) and for CUNY sentences in noise with adaptive SNRs (right panel). In the left panel, percent correct is measured and, therefore, the greater the scores the better the performance. In the right panel, reception threshold for sentences in $\mathrm{dB}$ SNR is measured, hence, the lower the number the better the performance. Average performance for the individual data and the ranges are compared for the experimental (Exp.) and everyday (Eday.) MAPs. Across-subject means (+SD) are shown in the right-hand portion of each panel. An asterisk represents a statistically significant difference between the two MAPs. Subject data are arranged left to right based on their performance in HINT sentences in quiet and CUNY sentences in noise, with the best performing subject on the left and the worst performing subject on the right.
0.05). Follow-up post hoc pairwise $t$ tests showed that scores of vowel discrimination continued to improve with increasing SNR $(\mathrm{p}<0.01)$ up to $+10 \mathrm{~dB}$. There were no significant differences in scores obtained with SNRs of +15 and $+10 \mathrm{~dB}$ and those obtained in quiet and SNR of $+15 \mathrm{~dB}(\mathrm{p}>0.05)$, but better scores were obtained in quiet than those obtained with an SNR of $+10 \mathrm{~dB}(\mathrm{p}<$ $0.01)$.

Results of the sentence discrimination tests, HINT sentences in quiet (left panel) and CUNY sentences in noise (right panel), are shown in figure 6 . Speech scores from each test were separately subjected to similar analyses. For the HINT sentences in quiet, there was no statistically significant difference in scores between the two MAPs $[F(1,11)=0.051, p>0.05]$. On the other hand, results showed that subject performance on the CUNY RTS measure was significantly better with the experimental MAP than with the everyday MAP $[\mathrm{F}(1,11)=17.992$, $\mathrm{p}<0.01$ ], i.e. subjects achieved $50 \%$ correct at lower (more difficult) SNRs which was roughly an improvement of $2 \mathrm{~dB}$ SNR with the experimental MAP than with their everyday MAP.

As can be seen in figures 4 and 6, overall performance with the experimental MAP was variable across listeners such that some subjects received larger benefits from lis- tening with the experimental MAP than other subjects. To examine this trend, the data were subjected to further analysis. The absolute differences in performance between the two MAPs were calculated to denote the advantages of listening with the experimental MAP. To consistently represent the advantage as a positive number, the difference in performance on the CUNY measure was calculated as 'everyday - experimental', and the consonant as 'experimental - everyday'. These data were compared against listeners ASM for masked MDTs. As can be seen in figure 7 , a significant correlation was found between masked MDT ASM and the advantages of listening with the experimental MAP using CUNY $\left(r^{2}=0.37, \mathrm{p}<0.05\right)$ and consonant $\left(r^{2}=0.55, \mathrm{p}<0.01\right)$ measures. Specifically, listeners who exhibited overall poor temporal acuity showed larger advantages and those who exhibited overall good temporal acuity demonstrated less advantages for listening with the experimental MAP.

Additionally, given that this study is concerned with determining the effect of listeners' temporal acuity on overall performance, it was important to examine the relation between modulation sensitivity and speech recognition across listeners. For this purpose, a correlation was conducted to examine subjects' ASM for masked MDTs obtained with all of the stimulation sites available in their 


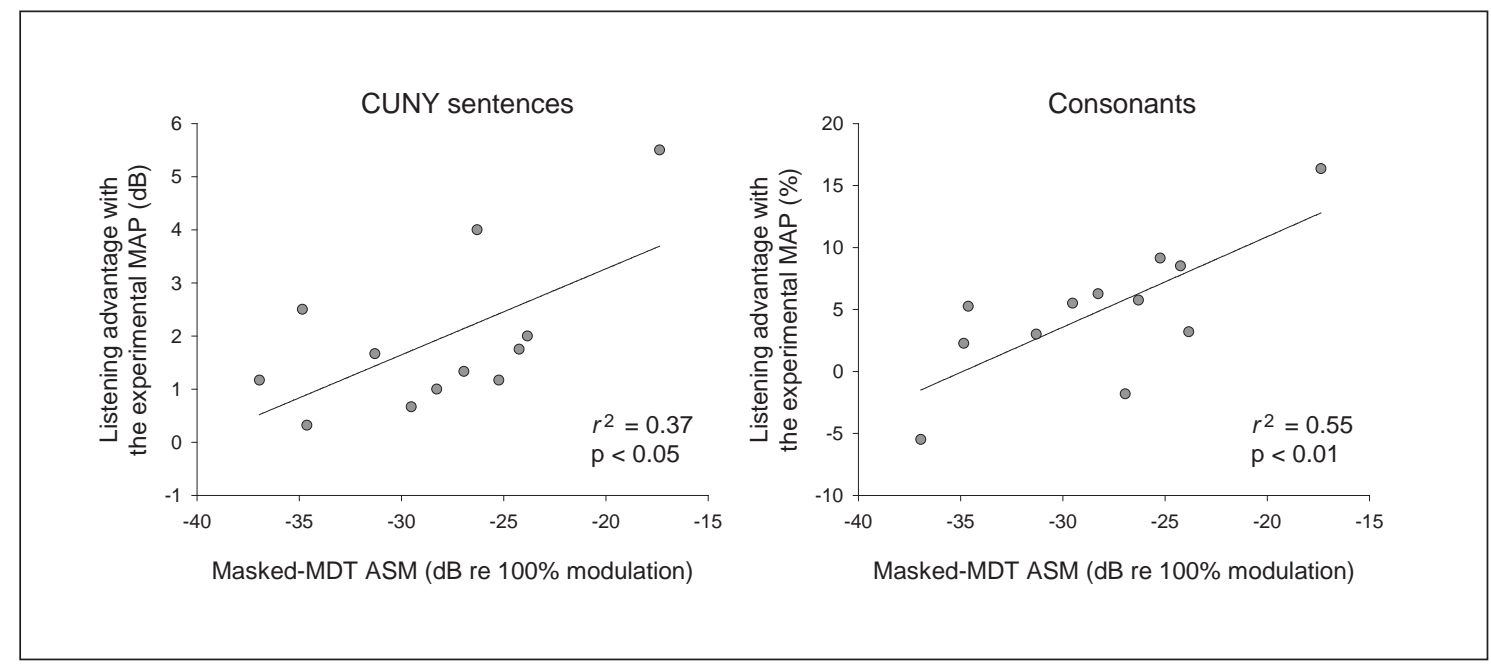

Fig. 7. Differences in performance between the everyday and the experimental MAPs calculated for both CUNY and consonant measures to derive the listening advantages with the experimental MAP. These differences are plotted against masked-MDT ASM. To display these advantages as a positive number, the difference in performance on the CUNY measure was calculated as 'everyday - experimental', and the consonant as 'experimental - everyday'.

electrode array and speech scores for consonants, vowels, HINT and CUNY measures. In general, the results of these analyses did not reveal a statistically significant correlation between listeners' average masked MDTs and their scores on HINT sentences, CUNY sentences in noise, vowels and consonants. However, a close examination of the relation between masked MDT and performance on the CUNY and consonant measures revealed a tendency for a positive relationship such that speech recognition for these measures improved with increased overall modulation sensitivity. This relationship was not significant for either measure $\left(r^{2}=0.12, \mathrm{p}>0.05\right.$ and $r^{2}=$ $0.06, p>0.05$, respectively), mainly because of two outlying data points (S52 and S77), see figure 8. An interquartile range test was used to examine for normality of distribution which revealed that data corresponding to subjects S52 and S77 are outliers as they exceed one and one half times the interquartile range. Therefore, these data points were removed and the analysis was repeated for all speech measures. A significant positive correlation with the masked MDTs was found for the CUNY data $\left(r^{2}=\right.$ $0.49, \mathrm{p}<0.05)$ and the consonant data $\left(r^{2}=0.47, \mathrm{p}<0.05\right)$ but not for other speech measures.

Variables that might have relevance to listeners' variability on overall modulation sensitivity and that could potentially serve as predictors of their performance were further examined in this study. Specifically, the factors of duration of deafness, age at onset of deafness and dura- tion of CI use were analyzed in relation to their ASM for masked MDT using regression analyses. Results did not reveal a significant relationship between these variables and masked MDTs, which suggests that these variables do not account for differences in modulation sensitivity among listeners.

\section{Discussion}

Problems with temporal resolution, channel interaction and other variables that depend on conditions near the implant electrodes often pose a challenge in electrical hearing and might result in less than desirable outcomes for some CI users. Hence, not all channels are equally efficacious for electrical stimulation. To address this problem, efforts should be focused on devising patient-specific mapping approaches that take into consideration differences in responses to electrical stimulation across the array of stimulation sites. By identifying and removing less optimal sites of stimulation, better speech recognition performance might be obtained. Based on this premise, the current study represented an initial step aimed at exploring the site-selection approach using a modulation sensitivity measure in the presence of a masker.

In order to identify differences in patterns of electrically elicited perception across sites of stimulation, MDTs were measured from all available sites across the electrode 


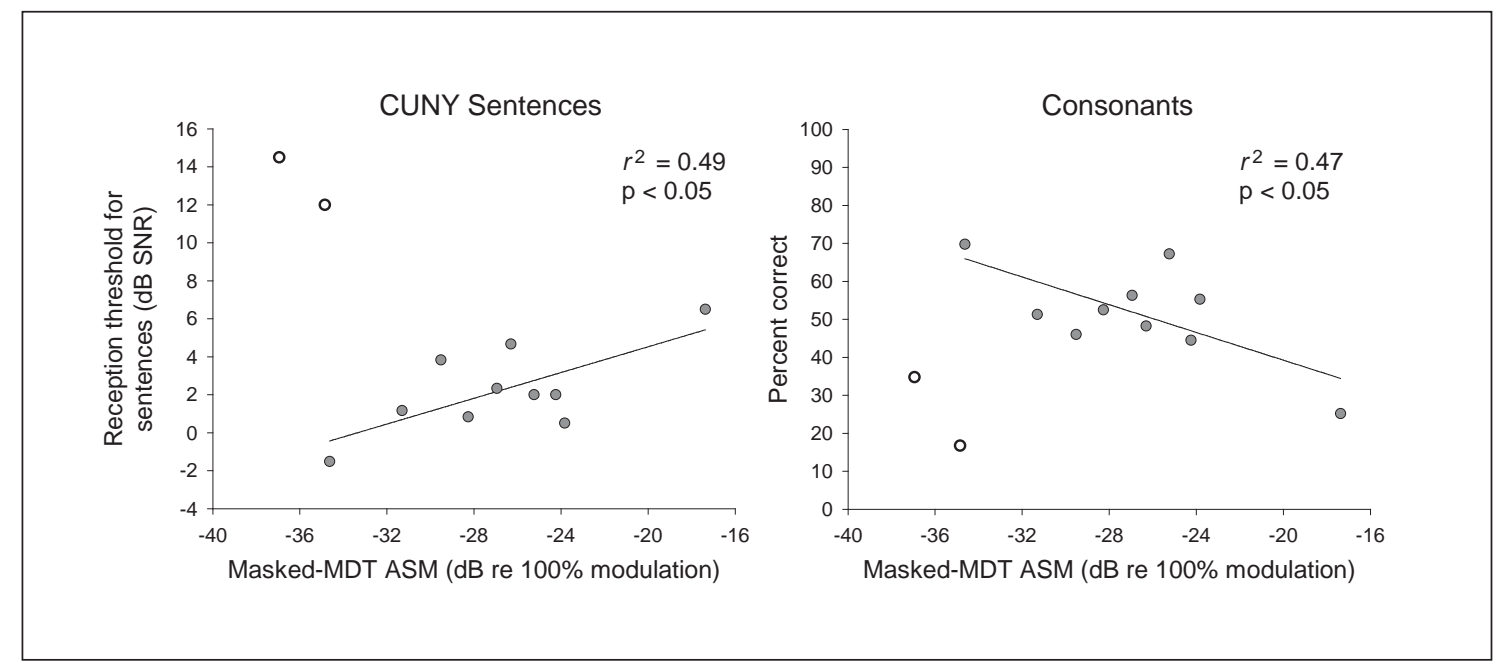

Fig. 8. ASMs for masked MDTs are compared against reception thresholds for CUNY sentences in noise (in the left panel) and against the averaged consonant scores (in the right panel). The open symbols in each panel represent the two data points that are considered as outliers based on the interquartile range test and, therefore, were not included in the regression analyses.

array in the presence of an unmodulated masker on an adjacent electrode. Site-to-site variability in response to electrical stimulation using the MDT measure can be seen in figure 2. As reported in the previous work by Garadat et al. [2012], patterns of modulation sensitivity were fairly variable within and across listeners. Generally, these results are in agreement with previous studies that examined MDTs in CI users [Fu, 2002; Colletti and Shannon, 2005; Pfingst et al., 2008].

This variation in MDT patterns is likely to be related to across-site variations in the conditions near the stimulation site such as the temporal properties of the activated neurons, the number of neurons near the stimulation site and other aspects of the electrode-neuron interface which could be altered due to the pathology of deafness as well as effects of the implantation. While these across-site variations in MDTs could also be mediated by central processes, this is less likely to be the case for the following reasons. The fact that across-site pattern of variation is subject specific [Pfingst et al., 2008; Garadat et al., 2012] suggests that the variation is not due to normal physiological variations or central processes, but rather it might be related to conditions created by deafness and implantation. Indeed, histological findings from deceased CI patients have demonstrated a wide range of pathological conditions along the cochlear spiral such as differences in nerve loss, myelination and the growth of fibrous tissue and new bone [Nadol et al., 1989; Fayad and Linthicum,
2006; Fayad et al., 2009]; these conditions vary along the cochlear spiral in subject-specific patterns and are likely to affect the temporal and spatial properties of the neural response to electrical stimulation in a site-specific manner. Other pathological conditions of the neurons might exist but not be evident under light-microscopic examination.

Within- and across-listener variability in modulation sensitivity could also be driven by differences in the important factors such as duration of CI use, duration of deafness and age at onset of deafness (table 1). For example, it has been reported that auditory deprivation produced by loss of hair cells can lead to a significant reduction in the activity of spiral ganglion neurons and eventually to a gradual neural degeneration [Liberman and Kiang, 1978; Hartmann et al., 1984; Shepherd and Javel, 1997]. The extent of this degeneration is likely to be nonuniform across sites and across patients. However, none of the analyses conducted showed a relation between these aforementioned factors and ASM or ASV across listeners. Taken together, these data suggest that variations in MDT patterns are more likely to be related to variation in local conditions near each electrode within and among listeners, and are not systematically predictable by any of the above-mentioned whole-subject variables examined in this study.

If electrical stimulation to sites with poor MDTs is eliminated, we expect that listeners' overall mean percep- 
tion of temporally modulated stimuli will be enhanced. In $\mathrm{n}$-of- $\mathrm{m}$ speech processing strategies, stimulation is delivered from the $\mathrm{n}$ channels with the highest amplitude. By eliminating stimulation to sites with poor MDTs, sites with better MDTs would be selected and, hence, perception of temporally modulated stimuli would be enhanced, which might lead to improved speech recognition. Therefore, it might be important to improve overall modulation sensitivity across the electrode array to measure such a relation. To ensure that the experimental MAPs indeed had better overall modulation sensitivity, mean MDTs were calculated and compared for the experimental and everyday MAPs. As shown in figure 3, mean MDTs that were obtained with the experimental MAPs were better than those obtained with the everyday MAP as expected.

In agreement with the above argument, subject performance on some speech measures significantly improved once a few sites with poor MDTs were turned off (experimental MAP) in comparison to their performance when these sites were stimulated (everyday MAP). This improvement was only observed for measures of consonants and sentence recognition in noise as can be seen in figures 4 and 6 (right panel), respectively. However, examining the data shown in these two figures, one can see that not all subjects received similar listening advantages from the experimental MAP; some subjects received far more benefit than others. The correlation analysis shown in figure 7 reveals that the benefit from the experimental MAP was dependent on the listeners' overall modulation sensitivity (their ASMs). Specifically, those who exhibited poor temporal modulation sensitivity received larger benefit from the experimental MAP than those who exhibited better modulation sensitivity. These results suggest that using this site-selection approach would be more beneficial to CI users who have poor temporal acuity. These results further imply that temporal modulation sensitivity is important for certain aspects of speech recognition and that by improving the perception of temporally modulated stimuli, speech recognition should also improve.

On the other hand, listener performance on the measure of vowel recognition was better with their everyday MAP than with the experimental MAP, indicating that this scheme was not beneficial for vowel recognition and was rather detrimental to performance. In general, these results support previous findings, which showed that vowel recognition is more dependent on spectral information [Dorman et al., 1998; Fu et al., 1998; Shannon et al., 1998] while consonant recognition is more reliant on temporal cues [Kiefer et al., 2000; Nie et al., 2006]. Hence, since the everyday MAPs had more spectral channels and finer spectral resolution per channel than the experimental MAPs, it is not surprising that better vowel recognition was obtained with the everyday MAP. Additionally, this could be related to differences in frequency-to-electrode allocation between the two MAPs that could introduce changes in the distance of separation among vowel formants. The effect of frequency-to-electrode allocation on vowel discrimination has been demonstrated in several studies [Skinner et al., 1995; Fu and Shannon, 1999; McKay and Henshall, 2002]. Furthermore, it has been shown that best performance was achieved with frequency-to-electrode allocation that was most familiar to the listeners [Friesen et al., 1999] and in this case, listeners were most familiar with the clinical MAPs.

As shown in figure 6 (left panel), no differences in performance between the everyday MAP and the experimental MAP were observed for sentence recognition in quiet. These results could be explained by two factors. First, the lack of differences between the two MAPs could be attributed to the ceiling effect that is evident on this test for most of our subjects. A ceiling effect could result when speech recognition is measured at a fixed intensity level and percent correct is measured, as opposed to speech reception thresholds that use adaptive tracking to find the minimum recognizable speech signal. This ceiling effect probably obscured any potential improvement that could have been obtained with the experimental MAPs. Second, these results are consistent with previous observations that the effect of temporal modulation sensitivity is more salient in the presence of background noise where CI users are more challenged [Skinner et al., 1994; MullerDeile et al., 1995; Battmer et al., 1997; Stickney et al., 2004] .

Overall, the results of the current study suggest that improving perception of temporal information might facilitate improvements in speech recognition. Previous reports demonstrated a relation between speech recognition and temporal modulation sensitivity in CI users [Cazals et al., 1994; Fu, 2002; Colletti and Shannon, 2005; Luo et al., 2008]. In line with these studies, a significant correlation was also found between listeners' mean modulation sensitivity and their performance on CUNY sentences in noise and consonant recognition measures (fig. 8), but not with their performance on HINT sentences in quiet and the vowel recognition measure. This correlation was only significant after excluding the results of two subjects (S52 and S77) who were treated as outliers based on the interquartile range test. Across-subject comparisons between psychophysical data and speech recognition data are subject to confounding effects of other subject variables such as cognitive factors. The site-selection strategy used in the 
current experiments used a within-subject design which is less susceptible to these confounding variables and, thus, can give a clearer picture of the importance of the psychophysical measure under investigation.

Findings from the current research suggest that by improving subjects' ASM perception of modulated signals, their ability to discern the temporal features that are important for sentence and consonant discrimination is enhanced. The improvement of mean modulation sensitivity can be achieved by the site-removal approach as demonstrated in this study. The disadvantage of this approach, however, is that removal of electrodes from the processor MAP might impact the ability of the listener to perceive spectral cues that are important for vowel recognition. An alternative approach would be to improve modulation sensitivity on those poor sites by using a parameter adjustment approach similar to that used by Garadat and Pfingst [2011]. By using this approach, perception of temporally modulated signals could potentially be enhanced at those poor sites. However, the extent to which this improvement in the perception of a modulated stimulus would mostly overcome the adverse influence of peripheral factors on performance with a cochlear implant needs to be determined in future studies.

In general, the current work underlines the importance of identifying differences in response to electrical stimulation along the tonotopic axis. These differences are likely due to variations in patterns of pathology that are specific to each patient. Establishing clinically convenient measures that correlate to functional differences in electrical stimulation is crucial to the use of a site-selection strategy to optimize patient outcomes. In the current study, patterns of masked MDTs were examined in relation to $\mathrm{T}$ and $\mathrm{C}$ levels, as well as the DRs for each subject. Similar to the results reported by Pfingst et al. [2008], the relation between MDTs and each of those commonly used clinical measures was quite inconsistent across subjects. Given that the perception of modulated signals is fundamental to speech recognition, future studies will aim to explore additional clinically convenient measures that might correlate with the masked MDT measure in order to facilitate its application in the clinical domain.

\section{Conclusion}

Using the psychophysical measure of temporal modulation sensitivity in the presence of a masker, the current study demonstrated differences in electrical hearing across the electrode array. The across-site patterns of modulation sensitivity were listener specific. These data suggest that modulation sensitivity depends, in part, on subject-specific variations in local conditions along the tonotopic axis in the implanted cochlea. By removing selected sites that exhibited poor masked MDTs from the subject's processor MAP and reallocating frequencies to the remaining sites, listener performance was improved on sentences in noise and consonant discrimination measures but deteriorated for vowel discrimination measure. Interestingly, this improvement for consonants and sentences in noise was greater for listeners who exhibited poor MDTs than those who exhibited good MDTs, suggesting that this site-selection strategy is more beneficial for listeners with poor temporal acuity. The results further suggest that differences across sites of stimulation can adversely affect overall implantation outcome. Therefore, measures that capture these differences could be integrated in the clinical mapping and fitting procedures to the benefit of the patients.

\section{Acknowledgements}

We express appreciation to our dedicated implanted subjects for their cheerful participation in this research. We are also grateful to Thyag Sadasiwan for programming the psychophysics platforms, to Ning Zhou for providing programs for the speech tests, and Brady West at the University of Michigan Center for Statistical Consultation and Research for consultation on the data analysis. We would also like to thank Deborah Colesa, Jennifer Benson and Caroline Arnedt for helping with subjects' logistics and Chris Ellinger for technical support. This work was supported by NIH/ NIDCD grants R01 DC010786 to Bryan E. Pfingst, F32 DC010318 to Soha N. Garadat and P30 DC05188 to Jochen Schacht.

\section{References}

\footnotetext{
Battmer RD, Feldmeier I, Kohlenberg A, Lenarz T: Performance of the new Clarion speech processor 1.2 in quiet and in noise. Am J Otol 1997;18:S144-S146.

-Bierer JA: Threshold and channel interaction in cochlear implant users: evaluation of the tripolar electrode configuration. J Acoust Soc Am 2007;121:1642-1653.

Bierer JA, Faulkner KF: Identifying cochlear implant channels with poor electrode-neuron interface: partial tripolar, single-channel thresholds and psychophysical tuning curves. Ear Hear 2010;31:247-258.

Boëx C, Kos MI, Pelizzone M: Forward masking in different cochlear implant systems. J Acoust Soc Am 2003;114:2058-2065.
}

Site Selection Based on Modulation Sensitivity
Audiol Neurotol 2013;18:247-260 DOI: $10.1159 / 000351302$ 
Cazals Y, Pelizzone M, Saudan O, Boex C: Lowpass filtering in amplitude modulation detection associated with vowel and consonant identification in subjects with cochlear implants. J Acoust Soc Am 1994;96:2048-2054.

-Chatterjee M: Modulation masking in cochlear implant listeners: envelope versus tonotopic components. J Acoust Soc Am 2003;113: 2042-2053.

-Chatterjee M, Shannon RV: Forward masked excitation patterns in multielectrode electrical stimulation. J Acoust Soc Am 1998;103:25652572.

- Cohen LT, Richardson LM, Saunders E, Cowan RS: Spatial spread of neural excitation in cochlear implant recipients: comparison of improved ECAP method and psychophysical forward masking. Hear Res 2003;179:72-87.

Colletti V, Shannon RV: Open set speech perception with auditory brainstem implant? Laryngoscope 2005;115:1974-1978.

Dorman MF, Loizou PC, Fitzke J, Tu Z: The recognition of sentences in noise by normalhearing listeners using simulations of cochlear-implant signal processors with 6-20 channels. J Acoust Soc Am 1998;104:3583-3585.

-Eisen MD, Franck KH: Electrode interaction in pediatric cochlear implant subjects. J Assoc Res Otolaryngol 2005;6:160-170.

- Fayad JN, Linthicum FH Jr: Multichannel cochlear implants: relation of histopathology to performance. Laryngoscope 2006;116:1310-1320.

-Fayad JN, Makarem AO, Linthicum FH Jr: Histopathological assessment of fibrosis and new bone formation in implanted human temporal bones using 3D reconsruction. Otolaryngol Head Neck Surg 2009;141:247-252.

-Friesen LM, Shannon RV, Baskent D, Wang X: Speech recognition in noise as a function of the number of spectral channels: comparison of acoustic hearing and cochlear implants. J Acoust Soc Am 2001;110:1150-1163.

Friesen LM, Shannon RV, Slattery WH 3rd: The effect of frequency allocation on phoneme recognition with the nucleus 22 cochlear implant. Am J Otol 1999;20:729-734.

-Fu Q-J: Temporal processing and speech recognition in cochlear implant users. Neuroreport 2002;13:1635-1639.

Fu Q-J, Shannon RV: Effect of electrode configuration and frequency allocation on vowel recognition with the Nucleus-22 cochlear implant. Ear Hear 1999;20:332-344.

- Fu Q-J, Shannon RV, Wang X: Effects of noise and spectral resolution on vowel and consonant recognition: acoustic and electric hearing. J Acoust Soc Am 1998;104:3586-3596.

-Garadat SN, Pfingst BE: Relationship between gap detection thresholds and loudness in cochlear-implant users. Hear Res 2011;275:130138.

-Garadat SN, Zwolan TA, Pfingst BE: Across-site patterns of modulation detection: relation to speech recognition. J Acoust Soc Am 2012; 131:4030-4041.

-Gfeller K, Oleson J, Knutson JF, Breheny P, Driscoll V, Olszewski C: Multivariate predic- tors of music perception and appraisal by adult cochlear implant users. J Am Acad Audiol 2008;19:120-134.

-Hartmann R, Top G, Klinke R: Discharge patterns of cat primary auditory fibers with electrical stimulation of the cochlea. Hear Res 1984;13: 47-62.

-Hillenbrand J, Getty LA, Clark MJ, Wheeler K: Acoustic characteristics of American English vowels. J Acoust Soc Am 1995;97:3099-3111.

Jones GL, Ho W, Drennan WR, Rubinstein JT: Relationship between channel interaction and spectral-ripple discrimination in cochlear implant users. J Acoust Soc Am 2013;133: 425-433.

- Kiefer J, von IIberg C, Rupprecht V, HubnerEgner J, Knecht R: Optimized speech understanding with the continuous interleaved sampling speech coding strategy in patients with cochlear implants: effect of variations in stimulation rate and number of channels. Ann Otol Rhinol Laryngol 2000;109:1009-1020.

Levitt H: Transformed up-down methods in psychoacoustics. J Acoust Soc Am 1971;49:467477.

Liberman MC, Kiang NY-S: Acoustic trauma in cats: cochlear pathology and auditory-nerve activity. Acta Otolaryngol (Stockholm) 1978; 358:1-63.

Luo X, Fu QJ, Wei CG, Cao KL: Speech recognition and temporal amplitude modulation processing by Mandarin-speaking cochlear implant users. Ear Hear 2008;29:957-970.

McKay CM, Henshall KR: Frequency-to-electrode allocation and speech perception with cochlear implants. J Acoust Soc Am 2002;111: 1036-1044.

Muller-Deile J, Schmidt BJ, Rudert H: Effects of noise on speech discrimination in cochlear implant patients. Ann Otol Rhinol Laryngol Suppl 1995;166:303-306.

Munson B, Nelson PB: Phonetic identification in quiet and in noise by listeners with cochlear implants. J Acoust Soc Am 2005;118:26072617.

Nadol JB Jr, Young YS, Glynn RJ: Survival of spiral ganglion cells in profound sensorineural hearing loss: implications for cochlear implantation. Ann Otol Rhinol Laryngol 1989; 98:411-416.

- Nelson DA, Donaldson GS, Kreft HA: Forwardmasking spatial tuning curves in cochlear implant users. J Acoust Soc Am 2008;123:15221543.

Nie K, Barco A, Zeng FG: Spectral and temporal cues in cochlear implant speech perception. Ear Hear 2006;27:208-217.

Nilsson M, Soli SD, Sullivan JA: Development of the Hearing in Noise Test for the measurement of speech reception thresholds in quiet and in noise. J Acoust Soc Am 1994;95:1085-1099.

Pfingst BE, Burkholder-Juhasz RA, Xu L, Thompson CS: Across-site patterns of modulation detection in listeners with cochlear implants. J Acoust Soc Am 2008;123:1054-1062.

Pfingst BE, Xu L: Across-site variation in detection thresholds and maximum comfortable loudness levels for cochlear implants. J Assoc Res Otolaryngol 2004;5:11-24.

Richardson LM, Busby PA, Clark GM: Modulation detection interference in cochlear implant subjects. J Acoust Soc Am 1998;104: 442-452.

Rosen S: Temporal information in speech: acoustic, auditory and linguistic aspects. Philos Trans R Soc Lond B Biol Sci 1992;336:367373.

Shannon RV, Jensvold A, Padilla M, Robert ME, Wang X: Consonant recordings for speech testing. J Acoust Soc Am 1999;106:L71-L74.

-Shannon RV, Zeng FG, Kamath V, Wygonski J, Ekelid M: Speech recognition with primarily temporal cues. Science 1995;270:303-304.

-Shannon RV, Zeng FG, Wygonski J: Speech recognition with altered spectral distribution of envelope cues. J Acoust Soc Am 1998;104: 2467-2476.

-Shepherd RK, Javel E: Electrical stimulation of the auditory nerve. I. Correlation of physiological responses with cochlear status. Hear Res 1997;108:112-144.

-Skinner MW, Clark GM, Whitford LA, Seligman PM, Staller SJ, Shipp DB, Shallop JK, Everingham C, Menapace CM, Arndt PL, et al: Evaluation of a new Spectral Peak coding strategy for the Nucleus 22 Channel Cochlear Implant System. Am J Otol 1994;15:15-27.

Skinner MW, Holden LK, Holden TA: Effect of frequency boundary assignment on speech recognition with the SPEAK speech-coding strategy. Ann Otol Rhinol Laryngol 1995;166: 307-311

-Skinner MW, Holden LK, Whitford LA, Plant KL, Psarros C, Holden TA: Speech recognition with the Nucleus 24 SPEAK, ACE, and CIS speech coding strategies in newly implanted adults. Ear Hear 2002;23:207-223.

-Stickney GS, Zeng FG, Litovsky R, Assmann P: Cochlear implant speech recognition with speech maskers. J Acoust Soc Am 2004;116: 1081-1091.

Swanson B: Nucleus MATLAB Toolbox 3.02 Software User Manual. Lane Cove, Cochlear, 2004.

Throckmorton CS, Collins LM: Investigation of the effects of temporal and spatial interactions on speech-recognition skills in cochlear implant subjects. J Acoust Soc Am 1999;105: 861-873.

-Van Tasell DJ, Soli SD, Kirby VM, Widin GP: Speech waveform envelope cues for consonant recognition. J Acoust Soc Am 1987;82: 1152-1161.

Wilson BS, Dorman MF: Cochlear implants: a remarkable past and a brilliant future. Hear Res 2008;242:3-21.

Wilson BS, Finley CC, Lawson DT, Wolford RD, Eddington DK, Rabinowitz WM: Better speech recognition with cochlear implants. Nature 1991;352:236-238

Zwolan TA, Collins LM, Wakefield GH: Electrode discrimination and speech recognition in postlingually deafened adult cochlear implant subjects. J Acoust Soc Am 1997;102: 3673-3685. 